The INL is a

U.S. Department of Energy

National Laboratory

operated by

Battelle Energy Alliance

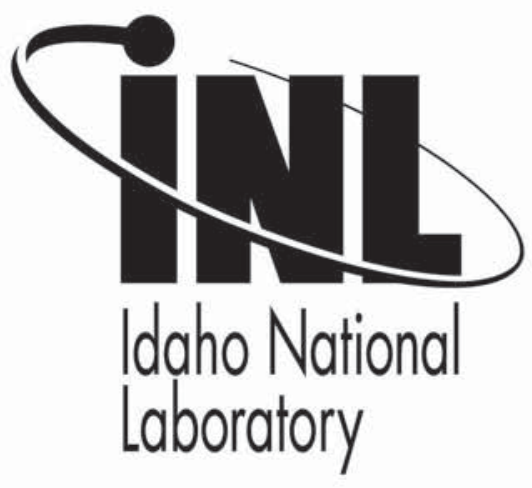

\title{
Recovery Discontinuous Galerkin Jacobian-Free Newton-Krylov Method for All-Speed Flows
}

\section{Fifth International Conference on Computational Fluid Dynamics (ICCFD5)}

HyeongKae Park

Robert Nourgaliev

Vincent Mousseau

Dana Knoll

July 2008

This is a preprint of a paper intended for publication in a journal or proceedings. Since changes may be made before publication, this preprint should not be cited or reproduced without permission of the author. This document was prepared as an account of work sponsored by an agency of the United States Government. Neither the United States Government nor any agency thereof, or any of their employees, makes any warranty, expressed or implied, or assumes any legal liability or responsibility for any third party's use, or the results of such use, of any information, apparatus, product or process disclosed in this report, or represents that its use by such third party would not infringe privately owned rights. The views expressed in this paper are not necessarily those of the United States Government or the sponsoring agency. 


\title{
Recovery Discontinuous Galerkin Jacobian-free Newton-Krylov Method for All-Speed Flows
}

\author{
HyeongKae Park, Robert Nourgaliev, Vincent Mousseau and Dana Knoll \\ Idaho National Laboratory Multiphysics Methods Group, \\ Idaho Falls, ID, 83415-3840, USA Ryosuke.Park@inl.gov \\ Robert.Nourgaliev@inl.gov Vincent.Mousseau@inl.gov Dana.Knoll@inl.gov
}

\section{Introduction}

There is an increasing interest to develop the next generation simulation tools for the advanced nuclear energy systems. These tools will utilize the state-ofart numerical algorithms and computer science technology in order to maximize the predictive capability, support advanced reactor designs, reduce uncertainty and increase safety margins. In analyzing nuclear energy systems, we are interested in compressible low-Mach number, high heat flux flows with a wide range of $R e, R a$, and $\operatorname{Pr}$ numbers. Under these conditions, the focus is placed on turbulent heat transfer, in contrast to other industries whose main interest is in capturing turbulent mixing. Our objective is to develop singlepoint turbulence closure models for large-scale engineering CFD code, using Direct Numerical Simulation (DNS) or Large Eddy Simulation (LES) tools, requireing very accurate and efficient numerical algorithms.

The focus of this work is placed on fully-implicit, high-order spatiotemporal discretization based on the discontinuous Galerkin method solving the conservative form of the compressible Navier-Stokes equations. The method utilizes a local reconstruction procedure derived from weak formulation of the problem, which is inspired by the recovery diffusion flux algorithm of van Leer and Nomura [VN05] and by the piecewise parabolic reconstruction [CW84] in the finite volume method. The developed methodology is integrated into the Jacobian-free Newton-Krylov framework [KK04] to allow a fully-implicit solution of the problem.

\section{Jacobian-free Newton-Krylov Framework}

The Jacobian-free Newton-Krylov (JFNK) [KK04] framework enables a fullycoupled solution of the nonlinear system. JFNK is a combination of quadratically convergent Newton's method and a Krylov subspace method [Saa03]. In each Newton iteration, the following linear system of equations is solved: 


$$
\begin{aligned}
J^{k} \delta \mathbf{U}^{k} & =-\operatorname{Res}\left(\mathbf{U}^{k}\right) \\
\mathbf{U}^{k+1} & =\mathbf{U}^{k}+\delta \mathbf{U}^{k}
\end{aligned}
$$

where, the superscript $k$ denotes the $k^{t h}$ Newton iterate. $J \equiv \frac{\partial R e s}{\partial U}$ is the Jacobian matrix and $\operatorname{Res}\left(\mathbf{U}^{k}\right)$ denotes the nonlinear residual functions. Computation of the Jacobian matrix can be prohibitively expensive. The JFNK method eliminates the direct computation of the Jacobian matrix by taking advantage of the fact that Krylov methods require only matrix-vector product to perform the iterations. To this end, the Jacobian-vector product is approximated by the forward finite difference of the form:

$$
J \mathbf{v} \approx \frac{\operatorname{Res}(\mathbf{U}+\varepsilon \mathbf{v})-\operatorname{Res}(\mathbf{U})}{\varepsilon}
$$

With this formulation, the linear system of equations can be solved by providing only subroutines for the nonlinear residual function evaluation. Thus, the JFNK framework provides a powerful platform to include a variety of nonlinear phenomena, especially useful for multiphysics simulations. This "Jacobianfree" version of Krylov methods can also be applied to the preconditioned system, which is the key to efficient numerical algorithm ${ }^{1}$. The Jacobian-vector product of the (right-) preconditioned system can be expressed as:

$$
J M^{-1} \mathbf{v} \approx \frac{\operatorname{Res}\left(\mathbf{U}+\varepsilon M^{-1} \mathbf{v}\right)-\operatorname{Res}(\mathbf{U})}{\varepsilon}
$$

\section{Recovery Discontinuous Galerkin Method}

The "recovery" discontinuous Galerkin ( $\mathrm{rDG}$ ) method was first introduced by van Leer and Nomura [VN05] for a diffusion operator. Their idea was to approximate a diffusion flux at cell edges by a higher-order reconstruction of the solution. As we found out, the concept of "recovery" can be extended to the advection and reaction operators. The $\mathrm{rDG}$ for the advection operator may be considered as the generalization of the Piecewise Parabolic finite volume method (PPM) [CW84]. In rDG, the higher-order solution within a cell is reconstructed by utilizing the solutions at the immediate neighboring cells. To do so, we first define the "recovered" function in cell $i$ as:

$$
\tilde{\mathbb{U}}_{i}(x)=\sum_{n=0}^{R} \tilde{\mathbb{U}}_{i}^{n} \mathfrak{L}_{n}(x)
$$

where $\tilde{\mathbb{U}}_{i}^{n}$ is the coefficient of the $n^{t h}$-order recovered function $\tilde{\mathbb{U}}_{i}(x)$. The recovered function is the $R^{t h}$-order polynomial, where $R=3 p+2$, and $p$

\footnotetext{
${ }^{1}$ For recent developments of the physics-based preconditioning in nuclear reactor applications, see [PNM08]
} 
is the order of the original DG method. The coefficients are computed by enforcing the following weak formulations:

$$
\begin{gathered}
\int_{\mathcal{I}_{m}} \mathfrak{L}_{n}(x) \tilde{\mathbb{U}}_{m}(x) d x=\int_{\mathcal{I}_{m}} \mathfrak{L}_{n}(x) U_{m}(x) d x \\
m=i, i \pm 1 \text { and } n=0, \ldots, p
\end{gathered}
$$

where $U_{m}$ is the original DG solution in cell $m$. Eq. (5) produces the $(R+1) \times$ $(R+1)$ system of equations, which can be solved analytically. Since the weak statements are used in reconstruction, the procedure is conservative and the first $p$-moments of the recovered coefficients are equal to those of the original DG solution. The advantage of the $\mathrm{rDG}$ is that the higher-order corrections are used only to compute the numerical fluxes at cell edges or integral reaction terms which appears upon DG discretization. As a result, it does not increase the number of unknowns of the solved nonlinear system.

Two-dimensional extension of rDG The recovery procedure discussed above can be extended to two-dimensional problems. The lowest order discretization $r D G_{0}(=\mathrm{PPM})$ uses the direction-by-direction recovery procedure; therefore it is only $1^{\text {st }}$ order in the cross derivatives. The recovered in-cell solution of the high-order rDG, on the other hand, includes high-order crossderivative terms, as well as normal derivatives. The key to this procedure is the choice of degrees of freedom (DoF) that avoids a null space. Fig. 1 shows our choice of DoF from neighboring cells in $r D G_{1}$, and Eq. (6) expresses the resulting recovered function.

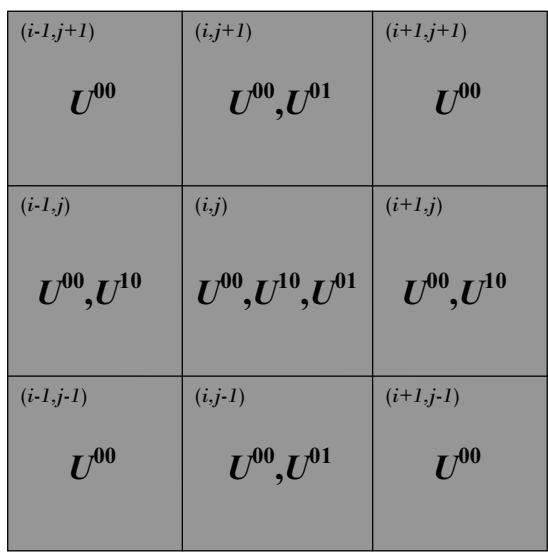

$$
U_{i, j}(x, y)=U_{i j}^{00}+U_{i j}^{10}+U_{i j}^{01}
$$

Fig. 1. The choice of the in-cell solution reconstruction for $r D G_{1}$ 


$$
\begin{aligned}
\tilde{\mathbb{U}} & =\tilde{\mathbb{U}}^{00} \mathfrak{L}_{0}(x) \mathfrak{L}_{0}(y) \\
& +\tilde{\mathbb{U}}^{10} \mathfrak{L}_{1}(x) \mathfrak{L}_{0}(y)+\tilde{\mathbb{U}}^{01} \mathfrak{L}_{0}(x) \mathfrak{L}_{1}(y) \\
& +\tilde{\mathbb{U}}^{20} \mathfrak{L}_{2}(x) \mathfrak{L}_{0}(y)+\tilde{\mathbb{U}}^{02} \mathfrak{L}_{0}(x) \mathfrak{L}_{2}(y)+\tilde{\mathbb{U}}^{11} \mathfrak{L}_{1}(x) \mathfrak{L}_{1}(y) \\
& +\tilde{\mathbb{U}}^{30} \mathfrak{L}_{3}(x) \mathfrak{L}_{0}(y)+\tilde{\mathbb{U}}^{03} \mathfrak{L}_{0}(x) \mathfrak{L}_{3}(y)+\tilde{\mathbb{U}}^{12} \mathfrak{L}_{1}(x) \mathfrak{L}_{2}(y)+\tilde{\mathbb{U}}^{21} \mathfrak{L}_{2}(x) \mathfrak{L}_{1}(y) \\
& +\tilde{\mathbb{U}}^{40} \mathfrak{L}_{4}(x) \mathfrak{L}_{0}(y)+\tilde{\mathbb{U}}^{04} \mathfrak{L}_{0}(x) \mathfrak{L}_{4}(y)+\tilde{\mathbb{U}}^{22} \mathfrak{L}_{2}(x) \mathfrak{L}_{2}(y) \\
& +\tilde{\mathbb{U}}^{50} \mathfrak{L}_{5}(x) \mathfrak{L}_{0}(y)+\tilde{\mathbb{U}}^{05} \mathfrak{L}_{0}(x) \mathfrak{L}_{5}(y) \\
& +O\left(\Delta x^{6}, \Delta y^{6}, \Delta x^{3} \Delta y, \Delta x \Delta y^{3}\right)
\end{aligned}
$$

where the subscripts denote the order of polynomial in $\mathrm{x}$ - and $\mathrm{y}$-direction, respectively. From Eq. (6), it is clear that the reconstructed function is the $6^{\text {th }}$ order accurate in $\mathrm{x}$ - and $\mathrm{y}$-direction and the $4^{\text {th }}$ order accurate in crossderivative terms.

\section{Numerical Examples}

In this section, we demonstrate high-order convergence of the rDG by solving the one- and two-dimensional Navier-Stokes equations. As the first example, we use a one-dimensional manufactured solution [NTP08]. Fig. 2 (left) shows the density error vs. total number of DoFs, used for $r D G_{0-3}$. All spatial discretizations exhibit nearly theoretical convergence rates. Fig. 2 (right) shows the convergence of five fully implicit time integration schemes, which also demonstrates the expected convergence rates.
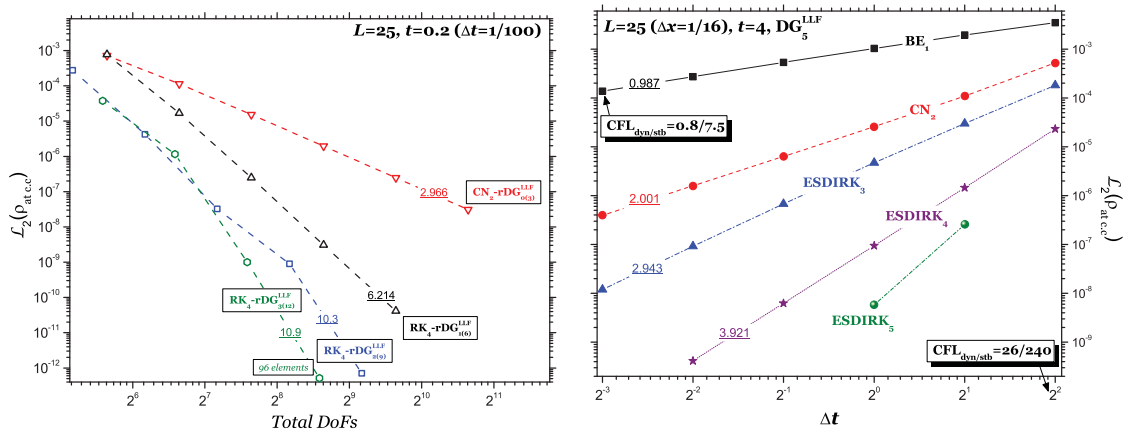

Fig. 2. Spatial (left), and temporal (right) convergence tests for $r D G_{0-3}$

The next example is the 2-D traveling wave problem, defined as

$$
\begin{aligned}
u(x, y, t) & =1+2 \cos (2 \pi(x-t)) \sin (2 \pi(y-t)) \\
v(x, y, t) & =1-2 \sin (2 \pi(x-t)) \cos (2 \pi(y-t)) \\
P(x, y, t) & =P_{0}-(\cos (4 \pi(x-t))+\cos (4 \pi(y-t)))
\end{aligned}
$$


Fig. 3 summarizes the convergence studies, comparing the $D G_{1}$ with $r D G_{1}$, for total energy and $\mathrm{x}$-momentum variables. Samples of the velocity and vorticity fields at $\mathrm{t}=0.0$ and $\mathrm{t}=0.15$ are shown at the bottom. As can be seen from Fig. 3, the convergence rates are the $2^{\text {nd }}$ and the $6^{\text {th }}$ order for $D G_{1}$ and $r D G_{1}$, respectively. A significant increase in accuracy is gained. Fig. 4 shows the efficacy (accuracy vs. CPU time), comparing the $D G_{1}$ with the $r D G_{1}$. Since the average number of the GMRES and Newton iterations does not increase in this problem, the CPU time solely depends on the number of nonlinear function evaluations. As one can see from Fig. 4, up to five orders of magnitude more accurate result can be obtained with same CPU time, On the other hand, to obtain the same accuracy, thr $r D G_{1}$ is $\approx 100$ times faster than the $D G_{1}$.
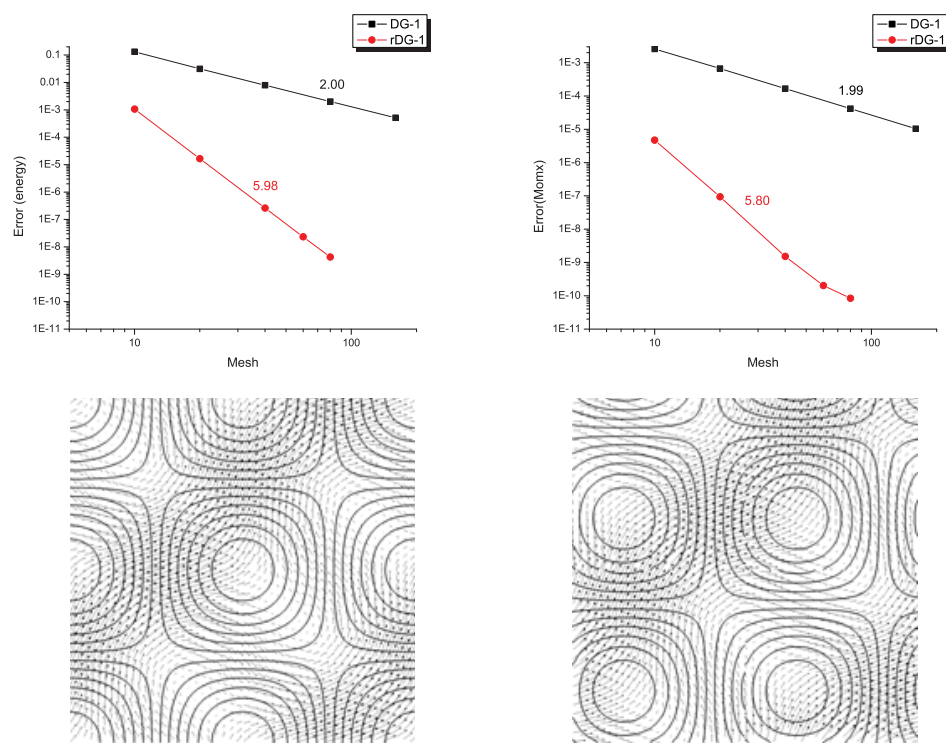

Fig. 3. Grid convergence of rDG for total energy (upper left), and x-momentum (upper right). Vorticity and velocity fields are shown at $\mathrm{t}=0.0$ (bottom left), $\mathrm{t}=0.15$ (bottom right)

\section{Conclusion}

In this work, we have developed very high-order spatial discretization scheme for hyperbolic and reaction operators based on the recovery of DG method. Numerical examples demonstrated nearly theoretical convergence for the smooth-flow tests. A significant saving in the computational time has been 


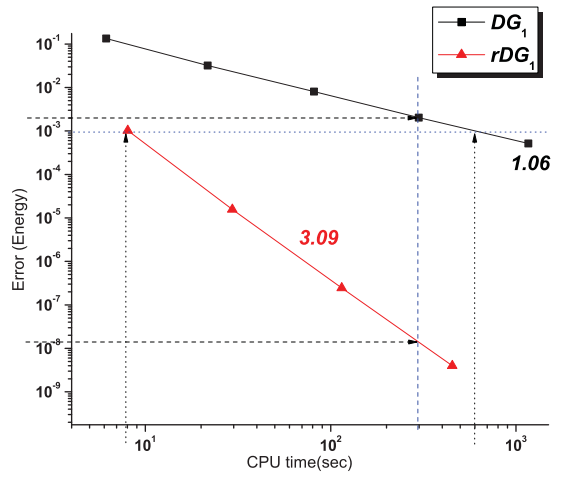

Fig. 4. Error vs. CPU time for the traveling-wave problem

achieved. The recovery procedure uses only the immediate neighbors to estimate higher order correction terms in the solution, which can be utilized for $a$ posteriori error indicator and subsequent mesh adaptivity. The next step is to extend the procedure to unstructured grids as well as to the three-dimensional problems.

Acknowledgment This manuscript has been authored by Battelle Energy Alliance, LLC under contract No. DE-AC07-05ID14517 (INL/CON-08-14501) with the U.S. Department of Energy. The United States Government retains and the publisher, by accepting the article for publication, acknowledges that the United States Government retains a nonexclusive, paid-up, irrevocable, world-wide license to publish or reproduce the published form of this manuscript, or allow others to do so, for United States Government purposes.

\section{References}

[KK04] Knoll, D. A., Keyes, D.: Jacobian-free Newton-Krylov Methods: A Survey of Approaches and Applications Journal of Computational Physics, 193, 357 (2004)

[CW84] Colella, P., Woodward, P. R.: The Piecewise Paraboilic Method (PPM) for Gas-Dynamical Simulations Journal of Computational Physics 54, 174 (1984)

[VN05] van Leer, B., Nomura, S.: Discontinuous Galerkin for Diffusion, 17th AIAA Computational Fluid Dynamics Conference Tronto, Ontario, Canada (2005)

[NTP08] Nourgaliev, R. R., et al.: Direct Numerical Simulation of Interfacial Flows: Implicit Sharp-Intrface Method (I-SIM), 46th AIAA Aerospace Sciences Meeting and Exhibit, Reno, Nevada, (2008)

[Saa03] Saad, Y.: Iterative Methods for Sparse Linear Systems, SIAM, philadelphia, 2nd edition (2003)

[PNM08] Park, H., et al.: Jacobian-free Newton-Krylov discontinuous Galerkin method and physics-based preconditioning for nuclear reactor simulations, International Conference on the Physics of Reactors (PHYSOR2008), Interlaken, Switzerland (2008) 